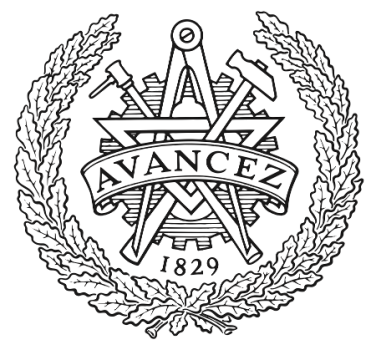

CHALMERS

UNIVERSITY OF TECHNOLOGY

\title{
Low kinetic inductance superconducting MgB2 nanowires with a 130-picosecond relaxation time for single-photon detection applications
}

Downloaded from: https://research.chalmers.se, 2023-04-26 14:02 UTC

Citation for the original published paper (version of record):

Cherednichenko, S., Acharya, N., Novoselov, E. et al (2021). Low kinetic inductance superconducting $\mathrm{MgB} 2$ nanowires with a 130-picosecond relaxation time for single-photon detection applications. Superconductor Science and Technology, 34(4). http://dx.doi.org/10.1088/1361-6668/abdeda

N.B. When citing this work, cite the original published paper. 
PAPER • OPEN ACCESS

Low kinetic inductance superconducting $\mathrm{MgB}_{2}$ nanowires with a $130 \mathrm{ps}$ relaxation time for single-photon detection applications

To cite this article: Sergey Cherednichenko et al 2021 Supercond. Sci. Technol. 34044001

View the article online for updates and enhancements.

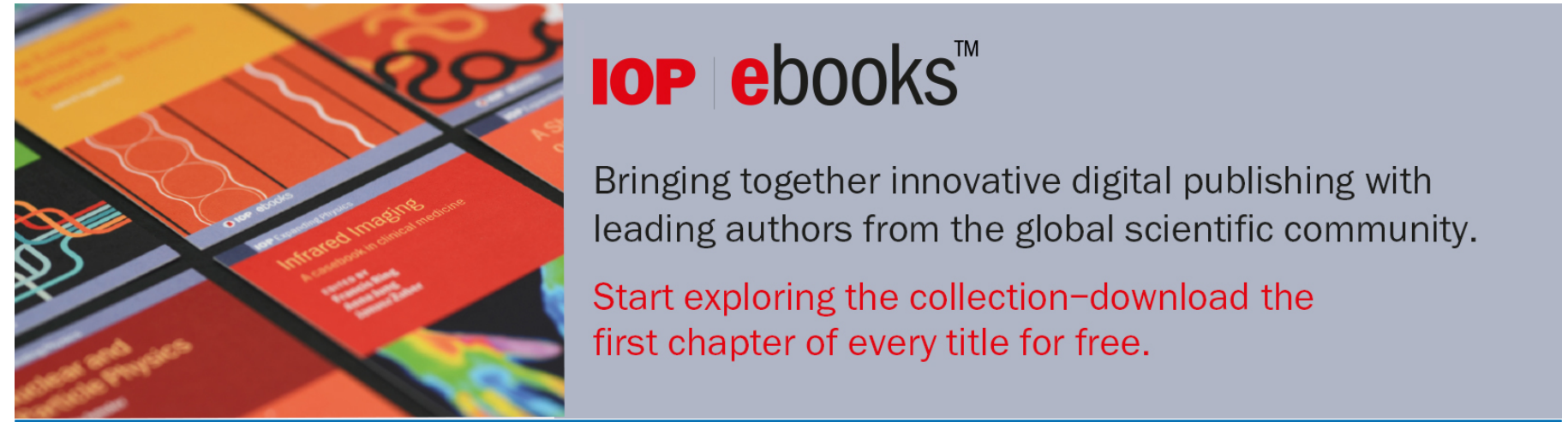




\title{
Low kinetic inductance
}

\section{superconducting $\mathrm{MgB}_{2}$ nanowires \\ with a 130 ps relaxation time for single-photon detection applications}

\author{
Sergey Cherednichenko $₫$, Narendra Acharya $₫$, Evgenii Novoselov $\mathbb{1}$ \\ and Vladimir Drakinskiy
}

Terahertz and Millimetre Wave Laboratory, Department of Microtechnology and Nanoscience, Chalmers University of Technology, SE-412 96 Gothenburg, Sweden

E-mail: serguei@chalmers.se

Received 29 October 2020, revised 21 December 2020

Accepted for publication 22 January 2021

Published 23 February 2021

\begin{abstract}
Properties of superconducting nanowires set the performance level for superconducting nanowire single photon detectors (SNSPDs). Reset time in commonly employed large area SNSPDs, 1-10 ns, is known to be limited by the nanowire's kinetic inductance to the load impedance ratio. On the other hand, reduction of the kinetic inductance in small area (waveguide integrated) SNSPDs prevents biasing them close to the critical current due to latching into a permanent resistive state. In order to reduce the reset time in SNSPDs, superconducting nanowires with both low kinetic inductance and fast electron energy relaxation are required. In this paper, we report on a study of kinetic inductance in narrow $(15-100 \mathrm{~nm})$ and long (up to $120 \mu \mathrm{m}$ ) superconducting $\mathrm{MgB}_{2}$ nanowires made from $5 \mathrm{~nm}$ thick films, offering such combination of properties. Such films were grown using hybrid physical chemical vapor deposition, resulting in a critical temperature of $\sim 32 \mathrm{~K}$, and a switch current density of $5 \times 10^{7} \mathrm{~A} \mathrm{~cm}^{-2}$ (at $4.8 \mathrm{~K}$ ). Using microwave reflectometry, we measured a kinetic inductance of $L_{\mathrm{k} 0}(4.8 \mathrm{~K})=1.3-1.6 \mathrm{pH} / \square$ regardless of the nanowire width, which results in a magnetic field penetration depth of $\sim 90 \mathrm{~nm}$. These values are very close to those in pristine $\mathrm{MgB}_{2}$. We showed that after excitations by a 50 fs pulsed laser the reset time in $35 \mathrm{~nm} \times 120 \mu \mathrm{m} \mathrm{MgB}_{2}$ nanowires is $130 \mathrm{ps}$, which is more than a factor of 10 shorter than in $\mathrm{NbN}$ nanowires of similar length-to-width ratios. Depending on the bias current, such $\mathrm{MgB}_{2}$ nanowires function as single-, double, or triple-photon detectors for both visible $(\lambda=630 \mathrm{~nm})$ and infrared $(\lambda=1550 \mathrm{~nm})$ photons, with a dark count rate of $<10 \mathrm{cps}$. Although the apparent photon detection efficiency seems so far to be low, further technological advances (uniform nanowire width, smaller thickness, increasing the switching current closer to the pair-breaking current) may improve this figure of merit.
\end{abstract}

Keywords: $\mathrm{MgB}_{2}$, kinetic inductance, superconducting nanowire, SNSPD, thin superconducting film, single photon detector

(Some figures may appear in colour only in the online journal) 


\section{Introduction}

During the last two decades we have seen enormous progress with superconducting nanowire single photon detectors (SNSPDs), from first demonstration of principles [1] to integrated circuits and a wide range of applications in nanophysics [2], high speed communication [3], quantum information [4], quantum key distribution [5], laser-ranging [6], deep space communication [7], etc. SNSPD's operation is based on photon assisted disruption of supercurrent in a superconducting nanowire, which is dc-biased close to the critical current $[8,9]$. Because the superconducting energy gap $\Delta$ is in the meV-range, a large number of non-equilibrium quasiparticles are generated at visible or infrared photon $(\sim \mathrm{eV})$ impacts. Both the small width of the nanowire, $w$ and the critical current, $I_{\mathrm{c}}$ being close to the pair-breaking current ensure high detection efficiency $[10,11]$. After hot-spot formation, the bias current is diverted from the SNSPD into the readout. Within a relaxation time, $\tau_{0}$ (set by electron-phonon non-elastic scattering and phonon escape into the substrate), non-equilibrium quasiparticles recombine into Cooper pairs. Until the supercurrent in the nanowire is restored and the hot-spot disappears (the reset time), SNSPD is blind to any further incoming photons. NbN SNSPDs, being the first one where singlephoton sensitivity was demonstrated, still provide the shortest reset time for a given detector area, compared to SNSPDs made from other superconductors. In $5 \mathrm{~nm}$ thick $\mathrm{NbN}$ films, the quasiparticle relaxation time $\tau_{0}$ is $\sim 30-50$ ps $[12,13]$. However, in long $(l)$ and narrow $(w) \mathrm{NbN}$ nanowires, large kinetic inductivity $\left(L_{\mathrm{k} 0}(4.8 \mathrm{~K})=80-90 \mathrm{pH} / \square\right)$ (inductance per square of the nanowire, $l=w$ ) leads to a significantly slower current return (restoration of the superconducting state), resulting in a much larger reset time $\tau$ [14]: $\tau=L_{\mathrm{k}} / R_{L} \gg \tau_{0}$, where $L_{\mathrm{k}}=L_{\mathrm{k} 0} \times l / \mathrm{w}$ is the total kinetic inductance of the nanowire and $R_{L}$ is the impedance of the read-out circuit $(\sim 50 \Omega)$. This is particularly critical in large area SNSPDs, with a kinetic inductance as large as hundreds of $\mathrm{nH}$, leading to a reset time of a few tens of ns. On the other hand, large kinetic inductance delays current return from the load $R_{L}$ back into the SNSPD, prior to the hot-spot cooling down, hence preventing the SNSPD from latching into a permanent normal (non-sensitive) state [15]. To a certain extent, the reset time can be reduced by choosing a high-impedance load. However, much increased $R_{L}$ leads to a reduction of the maximum nonlatching bias currents, hence precluding SNSPDs from reaching high detection efficiencies [16]. This problem is particularly pronounced in short SNSPD, where shunting resistors of small values or extra inductors in series are required to avoid latching, which does not altogether allow reaching short reset times $[17,18]$.

Apart from low $-T_{\mathrm{c}}$ superconductors $(\mathrm{NbN}, \mathrm{Nb}, \mathrm{NbTiN}$, TaN, MoN, WSi, MoSi, etc) [10, 15, 19-21], magnesium diboride $\left(\mathrm{MgB}_{2}\right)$ nanowires have also shown ability for single photon detection in both visible [22] and IR [23] ranges. However, the reset time in those $\mathrm{MgB}_{2}$ SNSPDs was reported to be 2-3 ns, i.e. close to that for NbN SNSPDs, the fact that was left unexplained. Previously it has been shown that in clean $\mathrm{MgB}_{2}$ (attainable in 50-200 nm thick films) the magnetic field penetration depth $\lambda_{0}$ is at least an order of magnitude shorter than that in $\mathrm{NbN}$ [24], which should lead to a small kinetic inductivity $\left(L_{\mathrm{k} 0}=\mu_{0} \lambda_{0}{ }^{2} / d\right)$. Apparently, due to a large number of defects in the abovementioned thin $\mathrm{MgB}_{2}$ films (normal state resistivity $\rho_{\mathrm{n}}>300 \mu \Omega \times \mathrm{cm}$ ) kinetic inductance increases, which affects the reset time. Recently, we managed to deposit $5 \mathrm{~nm}$ thick $\mathrm{MgB}_{2}$ films with a critical temperature $>30 \mathrm{~K}$ and $\rho_{\mathrm{n}}$ comparable to that in thick films. Furthermore, in such thin films, a quasiparticle relaxation time is measured to be $\tau_{0}=12 \mathrm{ps}$ [25], and which all together suggests that $\mathrm{MgB}_{2}$ nanowires might provide a solution for high speed photo detection.

In this work, we study both kinetic inductance and response rate in nanowires, with a large variation of width $(15-900 \mathrm{~nm})$ and length (3-120 $\mu \mathrm{m})$, made from clean (low normal state resistivity) and ultra-thin $(5 \mathrm{~nm}) \mathrm{MgB}_{2}$ films. Observing a kinetic inductance as low as $1.5 \mathrm{pH} / \square$, we show experimentally that a 130 ps voltage relaxation time can be achieved in $120 \mu \mathrm{m}$ - long devices into a standard $50 \Omega$ readout circuit with a $100 \mathrm{MHz}$ repetition rate. When biased close to the critical (switch) current, such devices show a linear dependence of the number of response pulses vs the incident laser power, corresponding to the single-photon detection regime.

\section{Film deposition and device fabrication}

$\mathrm{MgB}_{2}$ films were grown on $6 \mathrm{H}-\mathrm{SiC}$ substrates using a custombuilt hybrid physical chemical vapor deposition (HPCVD) system [26, 27]. The system was designed for a low deposition rate $\left(\sim 2.5-3 \mathrm{~nm} \mathrm{~min}{ }^{-1}\right)$ with a diborane gas flow of $2 \mathrm{sscm}$ $\left(5 \% \mathrm{~B}_{2} \mathrm{H}_{6}\right.$ in $\left.\mathrm{H}_{2}\right)$ and a deposition temperature of $700{ }^{\circ} \mathrm{C}$. As a result, continuous $\mathrm{MgB}_{2}$ films could be routinely obtained as thin as $5 \mathrm{~nm}$, as shown by transmission electron microscopy (TEM) (figure 1(a)), with a critical temperature of 32-34 K (figure 2(a)). Film growth on the $\mathrm{SiC}$ substrate is epitaxial, which ensures that bulk properties of $\mathrm{MgB}_{2}$ are preserved in the thin films as manifested in a fairly low normal-state (residual) resistivity of $\rho_{\mathrm{n}}=10-15 \mu \Omega \times \mathrm{cm}$, a high switch current density of $J_{\mathrm{c}} \sim 5 \times 10^{7} \mathrm{~A} \mathrm{~cm}^{-2}$, and a small magnetic field penetration depth of $\lambda=90 \mathrm{~nm}$ (see further in the text). TEM analysis was conducted on a set of $\mathrm{MgB}_{2}$ films of varying thickness, where the thickness proportionality to both the diborane flow and the deposition time was verified.

Subsequently, $\mathrm{MgB}_{2}$ films were transferred (vacuum broken) into a dc-magnetron sputtering system where plasma assisted cleaning of the film surface was done followed by in situ sputtering of a $20 \mathrm{~nm}$ thick Au film. This was followed by the device fabrication route \#1 which starts with defining the coplanar waveguide (CPW) contact pads and alignment marks on the $\mathrm{MgB}_{2}$ films with a $20 \mathrm{~nm}$ gold layer. A layer of polymethyl methacrylate (PMMA $-70 \mathrm{~nm}$ ) on top of copolymer (MMA-470 nm) was utilized as a positive tone bilayer resist system for electron beam lithography. The subsequent development in isopropanol/water mixture (10:1), electron beam physical vapor deposition of $\mathrm{Ti}-\mathrm{Au}-$ $\mathrm{Ti}(10 \mathrm{~nm}-200 \mathrm{~nm}-40 \mathrm{~nm})$, and lift-off in acetone leaves the desired patterns on the substrate. The first $10 \mathrm{~nm}$ titanium layer 


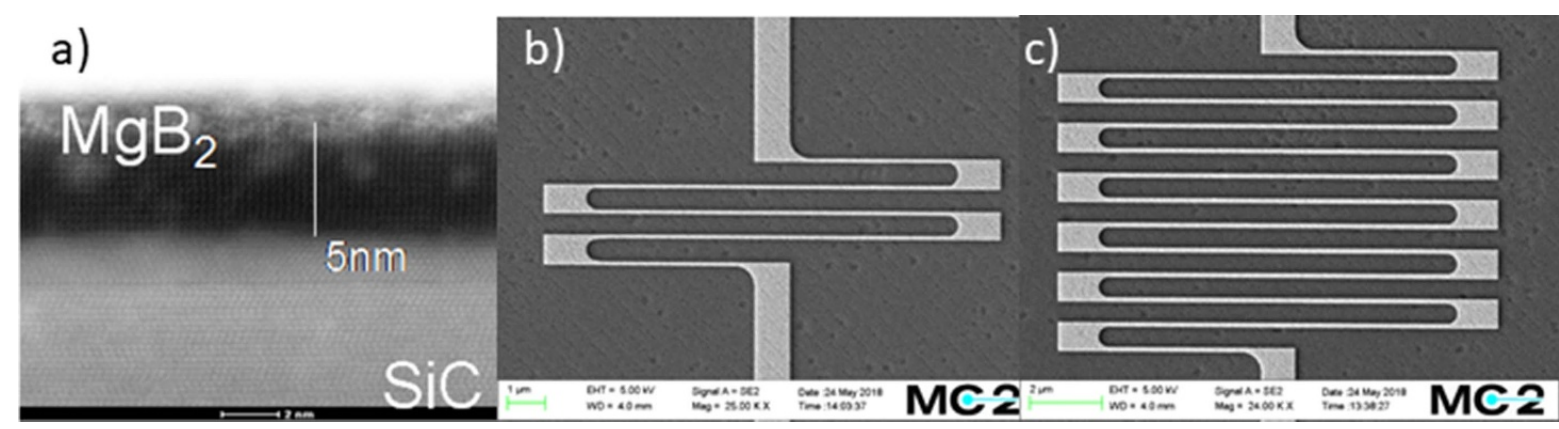

Figure 1. (a) TEM image of a $5 \mathrm{~nm}$ - thick $\mathrm{MgB}_{2}$ film on the $\mathrm{SiC}$ substrate. (b), (c) SEM images of a meandering $\mathrm{MgB}_{2}$ nanowires Scale bars are given in each of the images.

a)

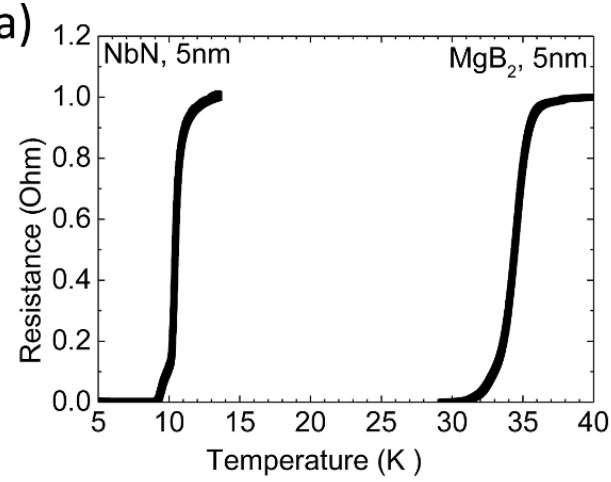

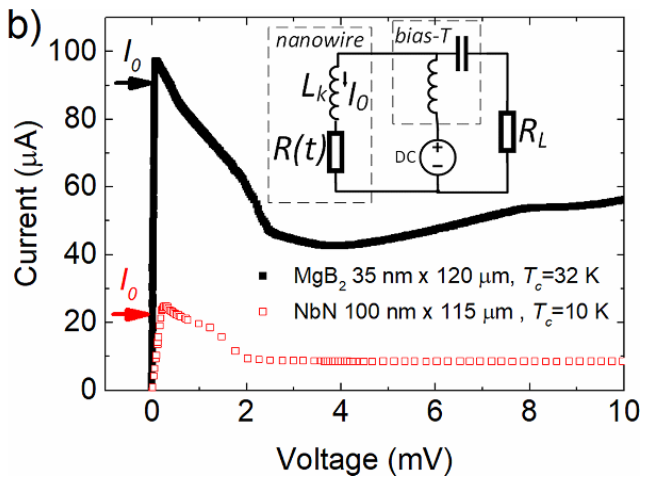

Figure 2. (a) Superconducting transitions (normalized resistance) in an $\mathrm{MgB}_{2}$ and an $\mathrm{NbN}$ nanowire. (b) $I(V)$-curves (at $5 \mathrm{~K}$ ) for a $\mathrm{MgB} 2$ and a $\mathrm{NbN}$ nanowire, utilized for the response rate experiments (see figure 5). Bias points are indicated with arrows. The inset shows simplified schematics of a photon detector (a variable resistance and an inductor, representing kinetic inductance in the nanowire) with a biasing network and a readout load (e.g. a low-noise amplifier).

promotes the adhesion of $\mathrm{Au}$ whereas the final $40 \mathrm{~nm}$ layer serves as a mask for gold contact pads later on during the $\mathrm{Ar}^{+}$ ion milling. The nanowire patterns were defined via another electron beam lithography step with a negative tone resist (maN 2401-100 nm), developed in tetramethlyamonium hydroxide aqueous solution (ma-D 525) followed by rinse in DI water. The remaining $\mathrm{MgB}_{2}$ and the initial $20 \mathrm{~nm} \mathrm{Au}$ layer, except on top the nanowire, were etched using $\mathrm{Ar}^{+}$ion milling. Finally, the negative resist mask layer was removed with acetone and the initial $20 \mathrm{~nm}$ Au layer on top of the nanowire was removed by another $\mathrm{Ar}^{+}$ion-milling step (figures 1(b) and (c)).

A second fabrication route has also been explored which differs from the route \#1 by absence of the initial gold protection layer (20 nm Au in Route \#1) on top of the $\mathrm{MgB}_{2}$ film. In this case, the final ion milling step, as in the case of route $\# 1$, is unnecessary, therefore there is no need in removing the negative resist mask and hence $\mathrm{Au}$ layer. The route \#2 contains fewer steps, whereas we have not observed any $\mathrm{MgB}_{2}$ film degradation due to absence of the protective Au layer.

In order to compare both the kinetic inductance and the reset time to those in a well-established material system, we have also fabricated and tested $100 \mathrm{~nm}$ wide $\mathrm{NbN}$ nanowires, both $115 \mu \mathrm{m}$ and $460 \mu \mathrm{m}$ long. The NbN film ( $d \sim 5 \mathrm{~nm}$ thick, as estimated from the deposition rate) was deposited by DC reactive magnetron sputtering in an $\mathrm{Ar}+\mathrm{N}_{2}$ environment on a
$330 \mu \mathrm{m}$ thick $C$-plane double side polished sapphire substrate maintained at $800{ }^{\circ} \mathrm{C}$. The film was patterned into $100 \mathrm{~nm}$ wide (100 nm spacing) meandering nanowires with reactive ion etching $\left(\mathrm{CF}_{4}: \mathrm{O}_{2}, 5: 1\right.$ flow ratio) through a mask defined by e-beam lithography on a positive (PMMA-80 nm) resist. During the RIE process, the total pressure was kept as low as possible (3.8 mTorr being the lowest used to provide a stable plasma discharge) in order to increase the etching anisotropy. Electrical contact pads (as single-port $50 \Omega \mathrm{CPW}$ ) were fabricated in $10 \mathrm{~nm} \mathrm{Ti} / 200 \mathrm{~nm} \mathrm{Au} / 40 \mathrm{~nm}$ Ti using a standard lift-off process. The NbN film between the CPW contacts was removed by means of wet-etching (nitric acid/hydrofluoric acid/acetic acid, 5:3:3) with a resistive mask placed over the nanowires (photo resist AZ1512). The critical temperature was $\sim 10 \mathrm{~K}$ and the room temperature sheet resistance was $\sim 300 \Omega / \square$, which corresponded fairly well to $\mathrm{NbN}$ film characteristics for previously published NbN SNSPDs.

\section{Experimental techniques}

All experiments were conducted in a cryogen-free probe station Lakeshore CRX-4K (base temperature $4.8 \mathrm{~K}$ ) with an optical view port and microwave ground-signal-ground probes on $X Y Z$ manipulator stages (figure 3(a)). Kinetic inductance was obtained from the imaginary part of 
the microwave impedance $\left(Z(f)=2 \pi \times f \times L_{\mathrm{k}}\right.$, where $f$ is the probing microwave frequency), which was measured using a (10 MHz-67 GHz) Vector Network Analyzer Rohde\&Schwarz ZVA 67. The vector network analyzer (VNA) calibration was done using a wafer with calibration standards (matched $(50 \Omega)$, open, and short) placed on the same cooling stage next to the $\mathrm{MgB}_{2}$ samples. Therefore, both the VNA calibration and impedance measurements of $\mathrm{MgB}_{2}$ nanowires were performed in a single cool-down. Magnetic (or geometric) inductance in superconducting films as thin as those utilized in our experiments is negligibly low, and it can be calculated analytically using the approach from [28] as a function of the film thickness $d$ and the temperature $T$ :

$$
\begin{aligned}
L_{\mathrm{m}}(d, T)= & \frac{\mu_{0} \lambda_{0}(T)}{4}\left[\operatorname{coth}\left(\frac{d}{2 \lambda_{0}(T)}\right)-\left(\frac{d}{2 \lambda_{0}(T)}\right)\right. \\
& \left.\cdot \operatorname{csch}\left(\frac{d}{2 \lambda_{0}(T)}\right)^{2}\right]
\end{aligned}
$$

where $\mu_{0}$ is the vacuum permeability and $\lambda_{0}(T)$ is the magnetic field penetration depth. Considering the $d=5 \mathrm{~nm}$ and $\lambda_{0}(5 \mathrm{~K})=90 \mathrm{~nm}, L_{\mathrm{m}}(5 \mathrm{~nm}, 5 \mathrm{~K})=0.5 \mathrm{fH} / \square$.

Lasers flood-illuminated the samples through a quartz pressure window of the probe station. The standard IR filter (not transparent for $1560 \mathrm{~nm}$ photons) on the $60 \mathrm{~K}$ shield was replaced with a WG12012-C window (Thorlabs). The output beam from the $\mathrm{cw} \lambda=630 \mathrm{~nm}$ laser diode module required no extra collimation, resulting in a light spot diameter of 3-4 mm. The IR ( $\lambda=1560 \mathrm{~nm}$, Toptica FemtoFErb 1560, $100 \mathrm{MHz}$ repetition rate) 50 fs pulse laser is fiber-coupled and a fiber launcher was utilized to collimate the IR laser beam. The IRlaser spot size (on the sample) was approximately $5 \mathrm{~mm}$, verified using SNSPD photo counts vs the laser $X Y$-shift. Two sets of light attenuators were utilized for the visible and IR light. The emission of both lasers ( $\sim 2 \mathrm{~mW}$ and $\sim 100 \mathrm{~mW}$ (average) for the $\lambda=630 \mathrm{~nm}$ and $\lambda=1560 \mathrm{~nm}$ lasers, respectively) was attenuated to a level where suppression of the switch current in the nanowires is not visible anymore.

At room temperature, both microwave readout and dc biasing were arranged (figure 3(b)) though a bias-T (Picosecond $5547,12 \mathrm{kHz}-15 \mathrm{GHz}$ ) followed by a low noise amplifier (MITEQ 3D-0010025, 0.5-5 GHz) and either a real time oscilloscope (Keysight Infiniium 54854 DSO, 4 GHz, $20 \mathrm{GSa} \mathrm{s}^{-1}$ ) or a pulse counter (HP 53131A 225). Nanowires were biased with a Yokogawa 7651 programmable dc source.

\section{Kinetic inductance}

As measured in a large variety of samples (width, length and fabrication batches), the kinetic inductivity in thin $\mathrm{MgB}_{2}$ nanowires was $1.35-1.60 \mathrm{pH} / \square$ at $4.8 \mathrm{~K}$ (figure 4(a)). In the case of defect-free uniform films, the total kinetic inductance $L_{\mathrm{k}}=L_{\mathrm{k} 0} \frac{l}{w}=L_{0} \frac{R}{R_{\mathrm{s}}}$ is expected to be proportional to the lengthto-width ratio $(l / w)$ and hence to the total resistance $R$ in the normal state $(40 \mathrm{~K}) . R_{\mathrm{S}}$ is the $\mathrm{MgB}_{2}$ film sheet resistance in the normal state. This trend was indeed observed (figure 4(b)), which confirms scalability of thin-film transport properties from the micron- to the nano-scales.

Kinetic inductance (and its temperature dependence) is a sensitive indicator for superfluid (Cooper pairs) density, hence providing the value for the superconducting energy gap. In $\mathrm{MgB}_{2}, \pi$ - and $\sigma$-sheets of the Fermi surface display quite dissimilar energy gaps $\Delta_{\pi}(T)$ and $\Delta_{\sigma}(T)$, with temperature dependences following [29]:

$$
\begin{aligned}
& \Delta_{\pi}(T)=\Delta_{\pi}(0) \Delta\left[1-\left(\frac{T}{T_{c}}\right)^{1.8}\right]^{\frac{1}{2}} \\
& \Delta_{\sigma}(T)=\Delta_{\sigma}(0) \cdot\left[1-\left(\frac{T}{T_{c}}\right)^{2.9}\right]^{\frac{1}{2}}
\end{aligned}
$$

$\Delta_{\pi}(0)$ and $\Delta_{\sigma}(0)$ are zero temperature energy gaps in $\pi$ - and $\sigma$-sheets.

Previously, in the frame of double-band modeling [30], it has been shown that electrodynamic properties in $\mathrm{MgB}_{2}$ can be analyzed by considering two quasi-layers (electrons of both $\pi$ - and $\sigma$-bands) in parallel, with a finite (yet, weak) interband scattering. In particular, partial contribution from each band to both the condensate energy and the superfluid density varies according to temperature and magnetic field, e.g. at zero magnetic field, the $\pi$-band is expected to contribute about $a>70 \%$ to the superfluid density $\left(\propto 1 / \lambda_{0}^{2}\right)$ at $T<\left(T_{\mathrm{c}}-2 \mathrm{~K}\right)$. The exact value of the coefficient $a$ depends on the interband scattering rate, and has to be evaluated for each particular case. However, the general trend seems to be correct, as in [24], where it was shown that $\lambda_{0} v s$ the mean free path can be modelled considering predominantly the $\pi$-band. In [31], a satisfactory agreement with experimental $\lambda_{0}(T)$ was demonstrated for thick films, considering two bands with an assumed $\pi$-band weighing coefficient $a=0.8$. For a general case of two conduction bands, the kinetic inductivity can be calculated from the imaginary part of the BCS conductivity [32] as:

$$
\begin{aligned}
L_{\mathrm{k} 0}(T)= & {\left[\left(\frac{\hbar \cdot 2 R_{\mathrm{S}}}{a \cdot \pi \cdot \Delta_{\pi}(T)} \cdot \frac{1}{\tanh \left(\frac{\Delta_{\pi}(T)}{2 k_{\mathrm{B}} \Delta T}\right)}\right)^{-1}\right.} \\
& \left.+\left(\frac{\hbar \cdot 2 R_{\mathrm{S}}}{(1-a) \cdot \pi \cdot \Delta_{\sigma}(T)} \cdot \frac{1}{\tanh \left(\frac{\Delta_{\sigma}(T)}{2 k_{\mathrm{B}} \cdot T}\right)}\right)^{-1}\right]^{-1}
\end{aligned}
$$

$k_{\mathrm{B}}$ is the Boltzmann constant, and $\hbar$ is the $2 \pi$-normalized Planck constant.

By fitting equation (2) to the experimental $L_{\mathrm{k} 0}(\mathrm{~T})$ data (figure 4(c)), we obtained the superconductor energy gaps at $T=0$. Our results showed that equation (1) holds even for extremely thin $\mathrm{MgB}_{2}$ films considering that $\Delta_{\pi}(0)=0.6 k_{\mathrm{B}} \cdot T_{\mathrm{c}}=1.7 \mathrm{meV}$ and $\Delta_{\sigma}(0)=2.2 k_{\mathrm{B}} \cdot T_{\mathrm{c}}=$ $6.2 \mathrm{meV}$, with an interband coefficient $a=0.85$. For a mean experimental value of $L_{\mathrm{k} 0}(5 \mathrm{~K})=1.5 \mathrm{pH} / \square$, we can obtain the effective magnetic field penetration depth: $\lambda_{0}$ 
a)

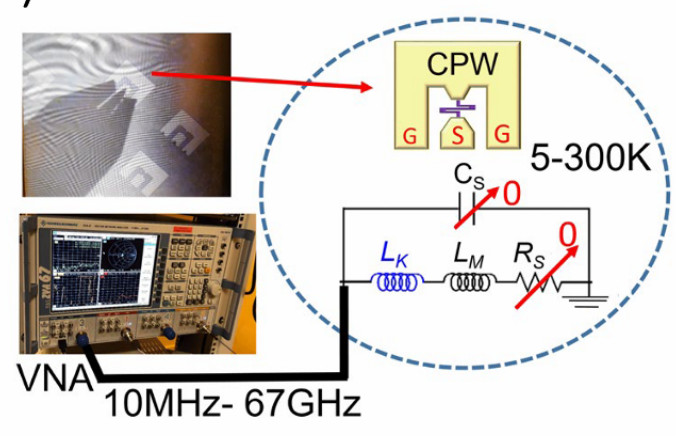

b)

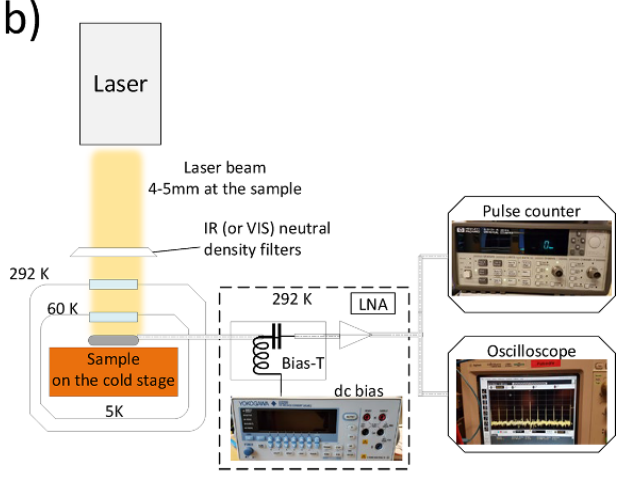

Figure 3. (a) The kinetic inductance measurements set-up. Chip layout (right), samples on the $5 \mathrm{~K}$ stage in the cryo-probe station with the microwave probe contacted (the view through the pressure window and the IR filter) (left-top), the VNA (left-bottom). (b) The optical characterization set-up. The laser flood illuminates the sample on the $5 \mathrm{~K}$ cooling stage through the window in the cryo probe-station.
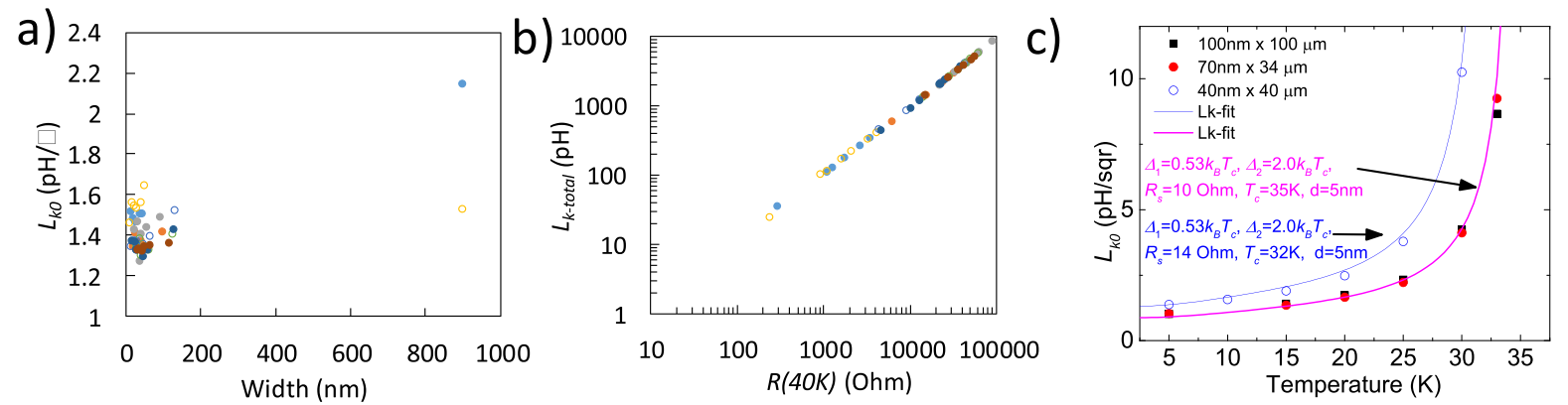

Figure 4. (a) Kinetic inductivity (at $4.8 \mathrm{~K}$ ) for samples with various widths and lengths. (b) The total kinetic inductance (at $4.8 \mathrm{~K}$ ) vs the normal state resistance $R(40 \mathrm{~K})$ for the same samples as in (a). (c) Kinetic inductivity vs temperature for nanowires from two batches with critical temperatures of $35 \mathrm{~K}$ and $32.7 \mathrm{~K}$. The fits are made using equation (2), where energy gaps for both $\pi$ - and $\sigma$-bands were calculated using equation (1) and the experimental $T_{\mathrm{c}}$.

$(5 \mathrm{~K})=\left(L_{\mathrm{k} 0} \times \mathrm{d} / \mu_{0}\right)^{1 / 2}=90 \mathrm{~nm}$. This value for the penetration depth for the measured resistivity of $\rho_{\mathrm{n}}=10$ $15 \mu \Omega \times \mathrm{cm}$ fits well with the $\lambda_{0}\left(\rho_{\mathrm{n}}\right)$-dependence previously reported for thick $\mathrm{MgB}_{2}$ films [24]. Increase of the penetration depth is related to a shorter electron mean free path $\ell$ vs the BCS coherence length $\xi_{0}$ as $\lambda_{0}(l, T)=\lambda_{L}(T)\left(1+\frac{\xi_{0}}{\ell}\right)^{\frac{1}{2}}$, where $\lambda_{L}(T)$ is the penetration depth in the clean limit [32]. The BCS coherence length can be estimated from $\xi_{0 \pi}=\hbar v_{\mathrm{F}} /\left(\pi \Delta_{\pi}\right)=66 \mathrm{~nm}$, considering the $a-b$ plane averaged Fermi velocity as $v_{\mathrm{F}} \sim 5.4 \times 10^{5} \mathrm{~m} \mathrm{~s}^{-1}$ [30]. For ultrathin films, as utilized in our study, electron scattering on the film surface might become significant (i.e. $\ell \sim 5 \mathrm{~nm}$ ), hence increasing both the residual resistivity and the penetration depth.

Small kinetic inductance in $\mathrm{MgB}_{2}$ nanowires is a very attractive feature in SNSPD applications. For one of the longest (in the number of squares, i.e. the $L / w$ ratio) of the utilized in our study $\mathrm{MgB}_{2}$ samples ( $35 \mathrm{~nm} \times 120 \mu \mathrm{m}, 3428$ squares) we measured its impulse response on excitations from a $50 \mathrm{fs}$ pulsed laser $(\lambda=1550 \mathrm{~nm})$. The measured total inductance for this sample was $5100 \mathrm{pH}$, which is expected to result in a voltage fall time of $\tau=L_{\mathrm{k}} / R_{L}=102$ ps for a $50 \Omega$ readout. The measured voltage impulses corresponded to the $100 \mathrm{MHz}$ repetition rate of the laser. The rise and the fall time of the nanowire response were 90 ps and 130 ps (10\%-90\%), respectively (figures 5(a) and (b)). The observed fall time is close to that calculated from the kinetic inductance.

For comparison, both the kinetic inductance and the impulse response were measured for two types of $100 \mathrm{~nm}$ wide $\mathrm{NbN}$ nanowires, $115 \mu \mathrm{m}$ (1150 squares) and $460 \mu \mathrm{m}$ (4600 squares) long. The kinetic inductance was measured in these samples (54 $\mathrm{nH}$ and $200 \mathrm{nH}$, respectively) using the same method as for $\mathrm{MgB}_{2}$ samples, corresponding to an average $L_{\mathrm{k} 0}(5 \mathrm{~K})=45 \mathrm{pH} / \square$. This value is $\sim 1 / 2$ of the previously reported [14], which can be due to variations of the $\mathrm{NbN}$ film quality (choice of the substrate, deposition conditions, etc), due to uncertainty in the film thickness, as well as due to variations of $L_{\mathrm{k}}$ measurements techniques. The voltage impulse decay time in $\mathrm{NbN}$ nanowires was $4.1 \mathrm{~ns}$ for the $460 \mu \mathrm{m}$ long sample and $1.3 \mathrm{~ns}$ for the $115 \mu \mathrm{m}$ long sample (figures 5(a) and (b)), both corresponding well to the measured kinetic inductance.

As follows from figures $5(\mathrm{a})$ and (b), $\mathrm{MgB}_{2}$ nanowires demonstrate a reset time $(10 \%-90 \%)$, which is at least a factor of 10 faster than in NbN nanowires of a similar geometry. Despite of being biased at the switch current, $\mathrm{MgB}_{2}$ nanowires with a kinetic inductance of $5 \mathrm{nH}$ demonstrate no latching, which follows from the fast decaying voltage impulses (figures 5(a) and (b)). Fast quasiparticles relaxation (12 ps in $\mathrm{MgB}_{2}$ vs $50 \mathrm{ps}$ in $\mathrm{NbN}$ ) [25] is a positive factor which reduces the effect of latching in small kinetic inductance samples. 

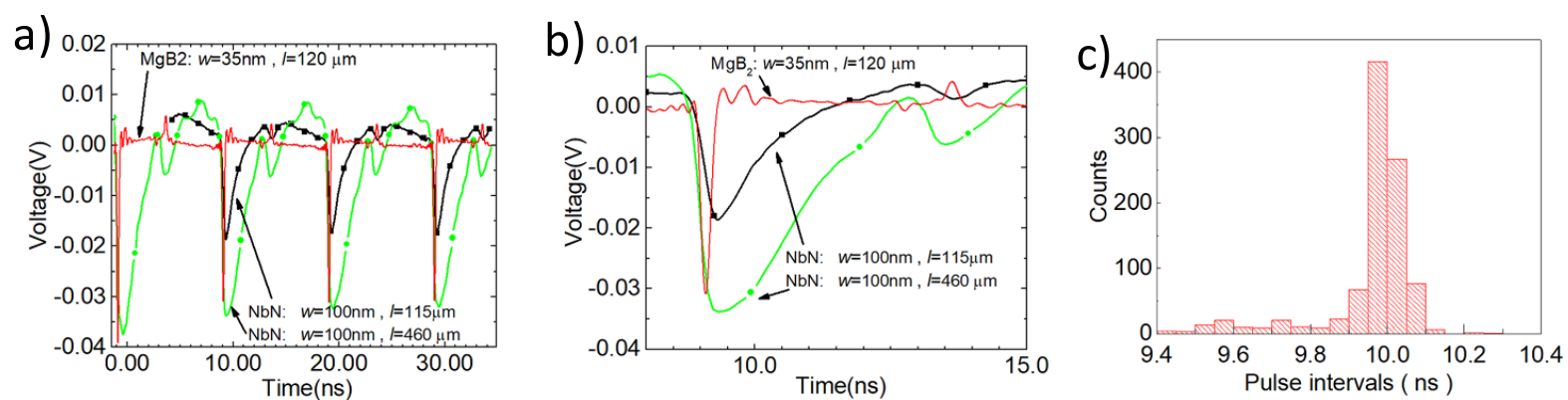

Figure 5. (a), (b) Voltage response to the IR pulsed laser (50 fs, $1560 \mathrm{~nm}, 100 \mathrm{MHz}$ repetition rate) of a $\mathrm{MgB}_{2}(35 \mathrm{~nm} \times 120 \mu \mathrm{m}$ (thin red, $\left.L_{\mathrm{k}}=5 \mathrm{nH}\right)$ sample and two $\mathrm{NbN}$ samples $\left(100 \mathrm{~nm} \times 115 \mu \mathrm{m}\right.$ (squares, $\left.L_{\mathrm{k}}=54 \mathrm{nH}\right)$ and $100 \mathrm{~nm} \times 460 \mu \mathrm{m}\left(\right.$ circles, $\left.\left.L_{\mathrm{k}}=200 \mathrm{nH}\right)\right)$ detectors with a total kinetic inductances (width and length variation). The same readout chain was used for all detectors $(0.05-5 \mathrm{GHz}$ $\mathrm{LNA}+4 \mathrm{GHz}$ oscilloscope). (c) Statistics for the response intervals (time span of $10 \mu \mathrm{s}$ ) for the $\mathrm{MgB}_{2}$ nanowire as in (a).
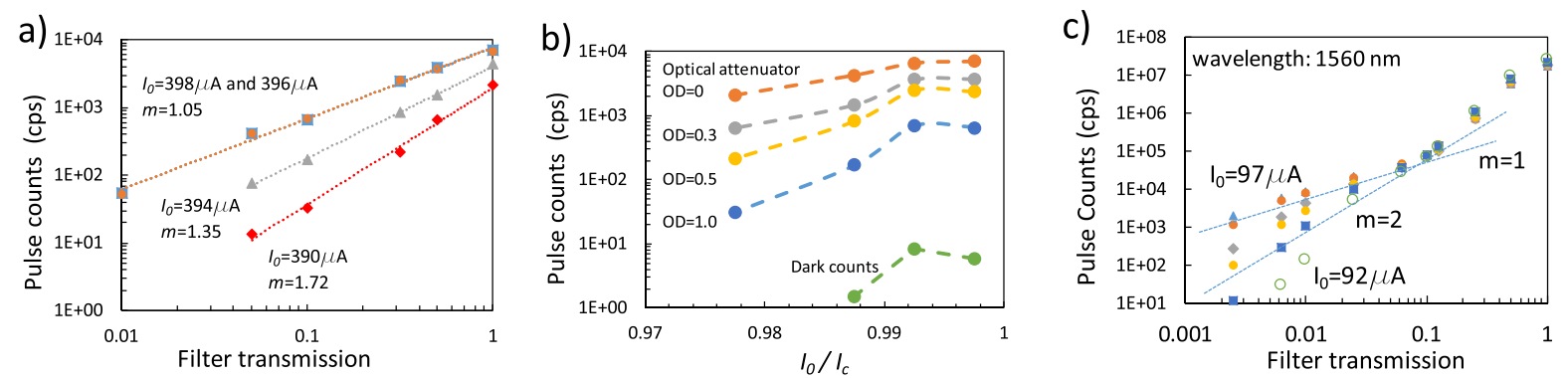

Figure 6. (a) Pulse counts vs relative photon flux (filter transmission with different optical densities OD). The $\mathrm{MgB}_{2} \mathrm{detector}$ $\left(90 \mathrm{~nm} \times 40 \mu \mathrm{m}, I_{\mathrm{c}}=399 \mu \mathrm{A}\right)$ was illuminated with a cw laser $(630 \mathrm{~nm})$. Lines are $N \propto F^{m}$ fits. (b) Pulse counts vs normalized bias current, for the same sample and the laser as in (a); (c)) Pulse counts vs photon flux for a $35 \mathrm{~nm}$ wide and $120 \mu \mathrm{m}$ long nanowire $\left(I_{\mathrm{c}}=97 \mu \mathrm{A}\right)$ illuminated with a pulsed laser $(50 \mathrm{fs}, 1560 \mathrm{~nm})$. Lines are $N \propto F^{m}$ fits. All measurements were made at $4.8 \mathrm{~K}$.

The voltage impulse rise dynamics in superconducting nanowire detectors has previously been studied both experimentally and theoretically in SNSPDs made from $\mathrm{NbN}$ $[14,33,34]$. One of the conclusions made there was that the rise time is proportional to the square root ratio of the total inductance and the normal domain resistance $\left(\sim \sqrt{\frac{L_{\mathrm{k}-\mathrm{tot}}}{R_{\text {spot }}}}\right)$, approaching nearly linear dependence at high inductance values $\left(\sim \frac{L_{k-\text { tot }}}{R_{\text {spot }}+R_{L}}\right)$. Because the sheet resistance in $\mathrm{NbN}$ is of the order of $500 \Omega$, the rise time in NbN SNSPDs is much less than the fall time. The sheet resistance in thin $\mathrm{MgB}_{2}$ films is $20-30 \Omega$, which can lead to a rise time longer than in $\mathrm{NbN}$ for the same total inductance. Considering that in $\mathrm{MgB}_{2}$ the kinetic inductance is a factor of 30-60 lower than in $\mathrm{NbN}$, the increased rise time will be noticed only for very long samples. Detailed modeling and experimental studies are needed in order to clear the question of hot-spot formation in $\mathrm{MgB}_{2}$ nanowires and to understand what will be the limiting factor for the rise time both for short and long samples.

For an initial estimate of the $\mathrm{MgB}_{2}$ photon detector response jitter, we utilized statistics for response pulse intervals collected within a $10 \mu$ s time-slot through a single oscilloscope scan, which was triggered by the first detection event. The response interval jitter is still $\sim 50 \mathrm{ps}$, which corresponds to a $\sim 25 \mathrm{ps}$ jitter of the rising edge (figure 5(c)). This value is an upper limit for $\mathrm{MgB}_{2}$ nanowire detectors, because a jitter less than that in $\mathrm{NbN}$ could be expected in $\mathrm{MgB}_{2}$ considering a factor of 33 lower kinetic inductance [33].

\section{IR- and visible light detection}

Direct demonstration of single photon detection capability requires a $100 \%$ photon coupling and a known quantum efficiency. Considering that the former is a challenging engineering task and the latter would require complex modelling, the single photon detection mode is often verified by statistical analysis of the pulse counts per photon flux [1] or inter-counts intervals [11]. We chose the first method, utilizing initially a cw laser $(630 \mathrm{~nm})$. The photon count rate $N$ vs the laser attenuation $F$ is shown in figure 6(a) at several bias currents for a $90 \mathrm{~nm}$ wide and $40 \mu \mathrm{m}$ long nanowire. For bias currents $I_{0} \geqslant 396 \mu \mathrm{A}$, the pulse count is linearly proportional to the photon flux, $N \propto F$. For smaller bias currents we observed that $N \propto F^{m}$, where $m>1$, which indicates that multiphoton detection mode becomes dominant. These results show that, in contrast to $\mathrm{NbN}$ nanowire detectors, the single photon detection mode is limited in $\mathrm{MgB}_{2}$ nanowires to a narrow bias current range in the vicinity of $I_{\mathrm{c}}$ (figure 6(b)). Physical modeling using accurate material parameters is required in order to obtain a full understanding of photon detection in $\mathrm{MgB}_{2}$ thin film nanowires. In this moment, we note that the aforementioned fact is probably caused by a smaller size of the normal domain $R_{\mathrm{ND}}$ (compared to the nanowire width), formed by the photon-deposited energy. Its order of magnitude could be estimated using an expression $R_{\mathrm{ND}}=\sqrt{\frac{E_{\mathrm{ph}}}{4 \pi \cdot d \cdot N(0) \cdot\left(k_{\mathrm{B}} \cdot T_{\mathrm{c}}\right)^{2}}}$ for a photon energy $E_{\mathrm{ph}}$ [9]. The total density of electronic 
states $N(0)$ at the Fermi level in $\mathrm{MgB}_{2}$ can be obtained e.g. from the electron specific heat data as $N(0)=\frac{3 \gamma_{\mathrm{n}}}{\pi^{2} k_{\mathrm{B}}^{2}}$. $\frac{\rho_{\mathrm{m}}}{M}=2.3 \times 10^{47} \mathrm{~m}^{-3} \mathrm{~J}^{-1}$, where $\gamma_{\mathrm{n}}=2.6 \mathrm{~mJ} \mathrm{~mol}^{-1} \mathrm{~K}^{-2}$ is the electron specific heat coefficient in the normal state [29], $\rho_{\mathrm{m}}=2.6 \mathrm{~g} \mathrm{~cm}^{-3}$ is the mass density, and $M=45.9 \mathrm{~g} \mathrm{~mol}^{-1}$ is the molar mass. The obtained $N(0)$ is close to that in $\mathrm{NbN}$ [35], whereas $T_{\mathrm{c}}$ in $\mathrm{MgB}_{2}$ is a factor of 3 higher. Therefore, a higher dc current (vs $I_{\mathrm{c}}$ ) is required to extend the normal domain to the edges of the nanowire. The fact that we have not observed any rise of the kinetic inductance at bias current close to the switch current most probably indicates that the switching current in our $\mathrm{MgB}_{2}$ nanowires is constriction-limited [36]. The observed dark count rate in the studied $\mathrm{MgB}_{2}$ nanowire detectors was very small, $<10 \mathrm{cps}$ at the highest utilized bias current $(398 \mu \mathrm{A})$, despite the fact that the samples were not shielded from either the electrical or magnetic field interference. At such bias current the dark count rates in NbN SNSPDs often reach values of $>10^{3}-10^{5} \mathrm{cps}[34,35]$. It is another indication that the switch current in our samples is limited by a constriction in a short section on the nanowire, leaving the most of the nanowire under-biased.

Similar results were obtained for detection of IR photons $(\lambda=1560 \mathrm{~nm})$ from the 50 fs pulsed laser by a $35 \mathrm{~nm} \times 120 \mu \mathrm{m} \mathrm{MgB} 2$ sample $\left(I_{\mathrm{c}}=97 \mu \mathrm{A}\right)$. For $I_{0}>0.97 \times I_{\mathrm{c}}$, a linear $N(F)$ dependence (figure 6(c)) indicates that the detector response is triggered by single photon absorption events. For $I_{0}<0.97 \times I_{\mathrm{c}}$, a combination of both one- and two-photon responses was observed $\left(N \propto F^{m}, m>1\right)$. At a high photon flux $(F>0.1)$, i.e. at a large number of photons per pulse, multi-photon response even dominates at the highest bias current, as has been observed in NbN SNSPDs [37] for large laser power.

\section{Conclusions}

In our study, we investigated ultra-thin $\mathrm{MgB}_{2}$ films in an attempt to create a new material basis for SNSPDs. Previously published works have demonstrated that single photon sensitivity is achievable with $\mathrm{MgB}_{2}$ nanowires. However, the reported reset times were $>1$ ns even for relatively short devices, which we interpret to result from a high degree of disorder in those films, manifested in both high normal state resistivity and high kinetic inductance. HPCVD was known to be a way of making clean $\mathrm{MgB}_{2}$ films, which so far been limited to thicknesses $>10-15 \mathrm{~nm}$. $\mathrm{MgB}_{2}$ films thinner than that were reported to break into a set of isolated islands (Volmer-Weber growth mode). Using our custom-built HPCVD system we have previously observed that by reducing the deposition rate to its lowest limit $\left(2.5-3 \mathrm{~nm} \mathrm{~min}^{-1}\right)$ $\mathrm{MgB}_{2}$ films as thin as $5 \mathrm{~nm}$ can be deposited, which allowed to fabricate $1 \mu \mathrm{m}^{2}$ devices (hot-electron bolometer, HEB, terahertz mixers) with a high switch current density. In the current paper, we show that even nanowires as narrow as $20 \mathrm{~nm}$ and with a large aspect ratio $(L / w>3000)$ can be fabricated using e-beam lithography and $\mathrm{Ar}^{+}$-ion milling using such $\mathrm{MgB}_{2}$ films. The obtained kinetic inductance is $\sim L_{\mathrm{k} 0}(4.8 \mathrm{~K})=1.3-$ $1.6 \mathrm{pH} / \square$ corresponding to a magnetic field penetration depth of $\lambda_{0}=90 \mathrm{~nm}$. Scalability of both the kinetic inductance and the normal-state resistivity from micrometer down to nanometer device scale indicates a high film uniformity. High quality of films is also manifested in a high critical temperature $(>30 \mathrm{~K})$, and a high switch current density $\left(\sim 5 \times 10^{7} \mathrm{~A} \mathrm{~cm}^{-2}\right)$, approaching some of the highest values ever achieved in $\mathrm{MgB}_{2}$ films. With the reset time being limited by kinetic inductance, $\mathrm{MgB}_{2}$ nanowire detectors are expected to operate a factor of $L_{\mathrm{k} 0}(\mathrm{NbN}) / L_{\mathrm{k} 0}\left(\mathrm{MgB}_{2}\right)=30-60$ times faster compared to $\mathrm{NbN}$ devices. We confirm it experimentally by observing a voltage impulse decay time of $130 \mathrm{ps}$ for an $\mathrm{MgB}_{2}$ nanowire with a total kinetic inductance of $5 \mathrm{nH}$, whereas this figure is $\sim 4 \mathrm{~ns}$ for an $\mathrm{NbN}$ nanowire with a similar $L / w$ ratio. Obtained results show that $\mathrm{MgB}_{2}$ nanowires are capable of single photon detection in both visible and communication $(1.5 \mu \mathrm{m})$ spectral ranges, combined with a low dark count rate, hence forming a technological platform for sensitive and high speed single photon detectors with a large cross-section and a low jitter. It also becomes apparent that the observed switching currents are probably limited by constrictions (a local narrowing), which prevents devices to be biased at high enough currents, hence reaching high detection efficiencies. This issues could be resolved by optimization of the nanowire fabrication process. At present conditions, considering the photon flux on the detector area, we estimate the detection efficiency as $\sim 10^{-2} \%$.

With the presently utilized deposition rate, the observed normal state resistivity in $\mathrm{MgB}_{2}$ films increases sharply whereas the critical temperature drops down as the film becomes thinner than $5 \mathrm{~nm}$. Nevertheless, it appears to be interesting to investigate if further reduction of the deposition rate would result in even thinner films $(2-3 \mathrm{~nm})$ with the desired degree of uniformity, low kinetic inductance, and high switch current density. Further steps shall also be taken towards increasing nanowire uniformity, i.e. avoiding constrictions and hence increasing the current density in the biased nanowire. We consider it important in order to reach a high quantum efficiency in SNSPDs made from $\mathrm{MgB}_{2}$ films.

\section{Acknowledgments}

The authors would like to thank: Dr Olof Bäcke for conducting TEM analysis of $\mathrm{MgB}_{2}$ samples; Professors X X Xi and $\mathrm{K}$ Chen for sharing their experience with the HPCVD process for $\mathrm{MgB}_{2}$ films and enlightening discussions; and Usman UlHaq for the help with process development. $\mathrm{MgB}_{2}$ film technology was developed under an ERC grant 308130-Teramix. This research was also supported by Swedish Research Council (Grant Nos. 2016-04189 and 2019-04345) and by Swedish National Space Agency (Grant No. 198/16). Devices were fabricated in the Nanofabrication Laboratory of Chalmers University of Technology.

\section{ORCID iDs}

Sergey Cherednichenko (D) https://orcid.org/0000-0002$5397-8024$ 
Narendra Acharya (D) https://orcid.org/0000-0003-2297-2321

Evgenii Novoselov (D) https://orcid.org/0000-0002-7971-

5927

Vladimir Drakinskiy (D) https://orcid.org/0000-0001-95193527

\section{References}

[1] Gol'tsman G N, Okunev O, Chulkova G, Lipatov A, Semenov A, Smirnov K, Voronov B, Dzardanov A, Williams C and Sobolewski R 2001 Appl. Phys. Lett. 79705

[2] Correa R E et al 2012 Nano Lett. 122953

[3] Robinson B S, Barron R J, Carney J J, Berggren K K, Kerman A J, Caplan D O, Yang J K, Hamilton S A, Stevens M L and Dauler E A 2006 Opt. Lett. 31444

[4] Hadfield R H 2009 Nat. Photon. 3696

[5] Takesue H, Nam S W, Zhang Q, Hadfield R H, Honjo T, Tamaki K and Yamamoto Y 2007 Nat. Photon. 1343

[6] Buller G S, Wallace A M, Nam S W, Hadfield R H, Hernandez-Marin S, Warburton R E and McCarthy A 2007 Opt. Lett. 322266

[7] Boroson D M, Robinson B S, Murphy D V, Burianek D A Khatri F, Kovalik J M, Sodnik Z and Cornwell D M 2014 Free Laser Commun. Atmos. Propag. XXVI 897189710 S

[8] Engel A and Schilling A 2013 J. Appl. Phys. 114214501

[9] Vodolazov D Y 2017 Phys. Rev. Appl. 7034014

[10] Lusche R, Semenov A, Ilin K, Siegel M, Korneeva Y, Trifonov A, Korneev A, Goltsman G, Vodolazov D and Hübers H W 2014 J. Appl. Phys. 116043906

[11] Marsili F, Najafi F, Dauler E, Bellei F, Hu X, Csete M, Molnar R J and Berggren K K 2011 Nano Lett. 112048

[12] Il'in K S, Lindgren M, Currie M, Semenov A D, Gol'tsman G N, Sobolewski R, Cherednichenko S I and Gershenzon E M 2000 Appl. Phys. Lett. 762752

[13] Zhang L et al 2018 Sci. Rep. 81486

[14] Kerman A J, Dauler E A, Keicher W E, Yang J K W, Berggren K K, Gol'tsman G and Voronov B 2006 Appl. Phys. Lett. 88111116

[15] Annunziata A J et al 2010 J. Appl. Phys. 108084507
[16] Kerman A J, Yang J K W, Molnar R J, Dauler E A and Berggren K K 2009 Phys. Rev. B 79100509

[17] Bitauld D, Marsili F, Gaggero A, Mattioli F, Leoni R, Nejad S J, Levy F and Fiore A 2010 Nano Lett. 102977

[18] Münzberg J, Vetter A, Beutel F, Hartmann W, Ferrari S, Pernice W H P and Rockstuhl C 2018 Optica 5658

[19] Schilling A, Engel A, Inderbitzin K, Hübers H-W, Semenov A, Siegel M, Hofherr M, Aeschbacher A and Il' in K 2012 Appl. Phys. Lett. 100062601

[20] Korneeva Y, Florya I, Vdovichev S, Moshkova M, Simonov N, Kaurova N, Korneev A and Goltsman G 2017 IEEE Trans. Appl. Supercond. 272201504

[21] Korneeva Y P et al 2014 Supercond. Sci. Technol. 27095012

[22] Shibata H, Takesue H, Honjo T, Akazaki T and Tokura Y 2010 Appl. Phys. Lett. 9798

[23] Shibata H, Akazaki T and Tokura Y 2013 Appl. Phys. Express 6023101

[24] Jin B B et al 2005 Supercond. Sci. Technol. 18 L1-L4

[25] Acharya N, Novoselov E and Cherednichenko S 2019 IEEE Trans. Terahertz Sci. Technol. 9565

[26] Xi X X et al 2007 Physica C 45622

[27] Novoselov E, Zhang N and Cherednichenko S 2017 IEEE Trans. Appl. Supercond. 277500605

[28] Meyerst N H and Member S 1961 Proc. IRE 491640

[29] Choi H J, Roundy D, Hong S, Cohen M L and Louie S G 2002 Nature 418758

[30] Eisterer M 2007 Supercond. Sci. Technol. 20 R47

[31] Kim M S, Skinta J A, Lemberger T R, Kang W N, Kim H J, Choi E M and Lee S I 2002 Phys. Rev. B 66645111

[32] Tinkham M 2004 Introduction to Superconductivity 2nd edn (Mineola: Dover Publications)

[33] Smirnov K V, Divochiy A V, Vakhtomin Y B, Sidorova M V, Karpova U V, Morozov P V, Seleznev V A, Zotova A N and Vodolazov D Y 2016 Appl. Phys. Lett. 109052601

[34] Nicolich K L, Cahall C, Islam N T, Lafyatis G P, Kim J, Miller A J and Gauthier D J 2019 Phys. Rev. Appl. 12034020

[35] Semenov A et al 2009 Phys. Rev. B 80054510

[36] Kerman A J, Dauler E A, Yang J K W, Rosfjord K M, Anant V, Berggren K K, Gol'tsman G N and Voronov B M 2007 Appl. Phys. Lett. 9010

[37] Elezov M S, Semenov A V, An P P, Tarkhov M A Goltsman G N, Kardakova A I and Kazakov A Y 2013 J. Opt. Technol. 80435 